\title{
Efeitos Genéticos e Ambientais que Afetam a Produção de Leite e Duração da Lactação de Cabras Mestiças no Estado da Paraíba
}

\author{
Edgard Cavalcanti Pimenta Filho1, José Lindenberg Rocha Sarmento², Maria Norma Ribeiro3, 4
}

RESUMO - Utilizaram-se dados de 689 lactações de 225 cabras Pardo Alpina x Gurguéia filhas de 18 bodes, controladas de 1988 a 1996, criadas na Fazenda Carnaúba, Taperoá-PB, com os objetivos de estudar os efeitos ambientais e estimar parâmetros genéticos para as características produção de leite (PL) e duração da lactação (DL). Os efeitos ambientais foram estimados por meio de um modelo estatístico que incluiu o efeito aleatório de bode, os efeitos fixos de grupo contemporâneo, tipo de parto e idade da cabra ao parto e duração da lactação, para PL, como covariáveis. Os parâmetros genéticos foram estimados por intermédio do programa MTDFREML, sob modelo animal, contendo os efeitos fixos e covariáveis utilizados no modelo anterior. As características foram influenciadas pelo efeito fixo de grupo contemporâneo. A idade da cabra influenciou apenas a característica PL. As médias obtidas para PL e DL foram $257,71 \pm 54,73 \mathrm{~kg}$ e 185,56 \pm 46,87 dias, respectivamente, equivalendo à produção média diária de 1,38 kg de leite/dia. As estimativas de herdabilidade e repetibilidade foram, respectivamente, de 0,23 e 0,35 para PL e 0,20 e 0,24 para DL. A correlação genética estimada entre as características foi de 0,96 . Em razão de as herdabilidades das características serem de média magnitude e limitadas ao sexo, sugerese o teste de progênie como método de seleção mais eficaz para os bodes.

Palavras-chave: caprinos leiteiros, nordeste, parâmetros genéticos, semi-árido

\section{Genetic and Environmental Effects that Affect Milk Production and Lactation Length of Crossbred Goats in the State of Paraiba}

\begin{abstract}
Records of 689 lactations of 225 Alpine x Gurguéia goats, progenies of 18 buck, controlled from 1988 to 1996 , raised at Carnaúba Farm, Taperoá-PB, were analyzed with the objective of study environmental effects and estimate genetics parameters for Milk Production (MP) and Length of Lactation (LL). The environmental effects were analyzed by an statistical model that included buck random effects and the fixed effects of contemporary groups, type of kidding and age of dam at kidding and length lactation as covariate. The genetic parameters were estimated using MTDFREML program, under animal model, including fixed effects and covariates through a described model before. The traits were influenced by contemporary groups. The age of dam at kidding influenced only MP trait. The means for MP and LL were $257.72 \pm 54.73 \mathrm{~kg}$ and $185.54 \pm 46.87$ days, respectively, giving a daily mean production of $1.38 \mathrm{~kg}$ of milk/ day. The heritability and repeatability estimates were, respectively, 0.23 and 0.35 for MP and 0.20 and 0.24 for LL. The genetic correlation estimate between MP and LL was 0.96 . Due to the traits heritability are of average magnitude and sex-limited, it is suggested to use the progeny test as method of more effective selection for bucks.
\end{abstract}

Key Words: genetic parameters, goats milk, Northeast, semi-arid

\section{Introdução}

O nordeste detém o maior rebanho caprino do país, com, aproximadamente, $93,7 \%$ do rebanho nacional. Em termos de tamanho populacional, a Paraíba é o quinto estado nordestino, com cerca de 403.801 cabeças, o equivalente a $6,1 \%$ da população caprina desta região. Quanto à densidade populacional, a Paraíba apresenta uma das maiores concentrações de caprinos do nordeste, destacando-se a microrregião do Cariri Ocidental, com densidade de $20 \mathrm{cabras} / \mathrm{km}^{2}$ (IBGE, 1996).
Apesar de numericamente expressivo, o rebanho caprino da região nordeste mantém índices produtivos ainda baixos, principalmente em razão do baixo padrão tecnológico empregado na região, que é fruto do sistema de produção adotado pela maioria dos criadores (cultura de subsistência) e da falta de apoio governamental.

A partir da iniciativa de alguns criadores dotados de maior visão empresarial, a caprinocultura leiteira vem se desenvolvendo na região e tem-se mostrado uma atividade promissora. Recentemente, o trabalho

\footnotetext{
${ }^{1}$ Professor do Departamento de Zootecnia/CCA/UFPB, 58397-000, Areia - PB. E.mail: edgard@cca.ufpb.br

2 Doutorando em Genética e Melhoramento. Bolsista da CAPES/UFV, Viçosa-MG. E.mail: lindeb@bol.com.br

3 Professora do Departamento de Zootecnia/UFRPE, Recife - PE. E.mail: mn.ribeiro@uol.com.br

${ }^{4}$ Bolsista do CNPq.
} 
integrado da Associação de Criadores de Caprinos do Rio Grande do Norte, amparado por efetivo apoio governamental, por meio do Programa do Leite, fez com que, em apenas três anos, a produção de leite caprino naquele estado alcançasse 2.200.000 litros anuais (Cordeiro, 2001).

Faltam ainda, no entanto, estudos que definam melhor os caminhos para a consolidação do negócio do leite caprino. $\mathrm{Na}$ área de melhoramento animal, fundamental para a sustentação do sistema de produção, pouco foi feito. A porcentagem de criadores que adotam a prática de controle leiteiro no Brasil e, especialmente, no Nordeste, é aquém do mínimo necessário para iniciar qualquer programa de seleção (Pimenta Filho et al., 2000). São escassas as estimativas de herdabilidade das características de produção de leite referentes à população regional, essenciais o melhor procedimento em programas de seleção. Dessa forma, os poucos trabalhos aqui realizados são referenciados com informações de outras regiões de clima tropical e temperado, havendo, então, a necessidade de realização de pesquisas na área de melhoramento genético com a população regional.

Estudos com raças nativas em regiões tropicais registraram valores de herdabilidade para produção de leite entre 0,15 e 0,35 (Mavrogenis et al., 1984; Constantinou et al., 1985). No Brasil, a maioria das pesquisas tem sido realizada com cabras originadas de clima temperado, como os trabalhos realizados por Gonçalves et al. (2001), com cabras das raças Saanen, Alpina e Toggenburg, que obtiveram estimativa de herdabilidade para produção de leite de $0,29 \pm 0,07$, e Soares Filho et al. (2001), que, ao trabalharem com as mesmas raças na região centro-oeste do país, encontraram herdabilidade de 0,$06 ; 0,12$ e 0,11 , respectivamente.

Embora a duração da lactação seja uma característica de produção, são poucos os autores que apresentam estimativas de sua herdabilidade: Ribeiro (1997), em animais da raça Saanen, verificou herdabilidade de 0,06 e Soares Filho et al. (2001), em cabras Saanen, Alpinas e Toggenburg, encontraram, respectivamente, 0,07; 0,07 e 0,03 de herdabilidade.

A correlação entre duas características de importância econômica deve ser estimada quando se deseja conhecer o grau de dependência entre as características. Essas estimativas, para caprinos, são escassas na literatura. As correlações genética e fenotípica estimadas por Kennedy et al. (1982) entre produção de leite e duração da lactação foram $0,78 \mathrm{e}$
0,66 respectivamente, para cabras das raças Alpina, Saanen e Toggenburg. Para essas raças, no Brasil, Gonçalves (1996) obteve 0,81 e 0,62 para as correlações genética e fenotípica, enquanto Ribeiro (1997) registrou 0,43 e -0,50, respectivamente, em rebanho Saanen.

Objetivou-se, com este estudo, analisar a influência de alguns efeitos de ambiente e estimar parâmetros genéticos para as características produção de leite e duração da lactação de caprinos mestiços criados no semi-árido paraibano, utilizando a metodologia da Máxima Verossimilhança Restrita sob modelo animal.

\section{Material e Métodos}

Foram utilizados dados referentes a lactações de cabras mestiças Pardo Alpina x Gurguéia, controladas no período de 1988 a 1998 na Fazenda Carnaúba, em Taperoá, Estado da Paraíba. A fazenda está localizada na microrregião do Cariri Ocidental, a $7^{\circ} 12^{\prime} 23^{\prime \prime}$ de latitude sul e $36^{\circ} 49^{\prime} 25^{\prime \prime}$ 'de longitude W.G., a $500 \mathrm{~m}$ de altitude.

O clima da região está incluído no subtipo B, subdesértico quente, de tendência tropical, com precipitação média de $300 \mathrm{~mm}$ anuais, e apresenta curta estação chuvosa, com estação seca prolongada (maior que oito meses) e umidade relativa do ar em torno de $65 \%$.

Os animais foram criados em sistema semiextensivo e alimentados com capim-buffel (Cenchrus ciliaris L.) e pasto nativo, na estação chuvosa, e capim-elefante (Pennisetum purpureum schum.), palma forrageira (Opuntia sp.), raspa de mandioca (Manihot sativa), bagaço de cana hidrolisado (Sacharum oficcinarum) e concentrado protéico, na estação seca, além de sal mineral à vontade. Utilizouse o sistema de monta natural controlada e controle sanitário, sistematicamente. O controle leiteiro foi realizado a cada 28 dias, em duas ordenhas diárias, com intervalo de 12 horas. A partir das fichas de produção e reprodução, foi editado um arquivo contendo o número da cabra, paternidade e maternidade, data de nascimento, datas dos partos, produção de leite, duração da lactação, tipo e ordem de parto e intervalo de partos.

Inicialmente, o arquivo continha 1.939 registros de produção de leite. Para gerar o arquivo utilizado nas análises, foram feitas algumas restrições, permanecendo no arquivo cabras paridas entre 1988 e 1996, animais com pai e mãe conhecidos, bodes com, no mínimo, cinco filhas no rebanho, grupos de contemporâneos com, no mínimo, dois animais (cabras

R. Bras. Zootec., v.33, n.6, p.1426-1431, 2004 
que produziram mais de 90 e menos de $500 \mathrm{~kg}$ de leite por lactação, com durações de mais de 70 e menos de 400 dias e máximo de 12 parições). Após os descartes, ficaram disponíveis para a análise 689 registros de produção de leite e duração da lactação de 255 cabras filhas de 18 reprodutores e 142 matrizes.

Oestudo dos fatores de meio que podem influenciar as características produção de leite (PL) e duração da lactação (DL) foi feito pelo método dos quadrados mínimos, por meio do PROC GLM do SAS ${ }^{\circledR}$ (1999), utilizando o comando RANDOM, que considera o efeito de reprodutor como aleatório, a partir do seguinte modelo estatístico:

$$
\begin{aligned}
& Y_{i j k l}=\mu+R_{i}+G C_{j}+T_{k}+b_{1}\left(I_{i j k l}-\bar{I}\right)+b_{2}\left(I_{i j k l}-\bar{I}\right)^{2}+ \\
& b_{3}\left(D L_{i j k l}-\overline{D L}\right)+b_{4}\left(D L_{i j k l}-\overline{D L}\right)^{2}+\varepsilon_{i j k l}
\end{aligned}
$$

em que: $Y_{i j k l}=\mathrm{PL}$ do animal 1, filha do bode i, pertencente ao grupo contemporâneo j, e com tipo de parição k; $\mu=$ média geral da população; $R_{i}=$ efeito aleatório do bode i; $G C_{k}=$ efeito fixo de grupo contemporâneo $\mathrm{k} ; T_{k}=$ efeito fixo de tipo de parto, sendo 1 correspondente ao parto simples e 2 ao múltiplo; $I_{i j k l}=$ efeito da idade da cabra ao parto, como covariável; $\bar{I}=$ média da covariável; $b_{1}=$ coeficiente de regressão linear para idade da cabra ao parto; $b_{2}=$ coeficiente de regressão quadrático para idade da cabra ao parto; $D L_{i j k l}=$ efeito da duração da lactação, como covariável; $b_{3}=$ coeficiente de regressão linear para duração da lactação; $b_{4}=$ coeficiente de regressão quadrática para duração da lactação; $\overline{\mathrm{DL}}=$ média da covariável; $\varepsilon_{i j k l}=$ erro aleatório independentemente distribuído com média zero e variância $\sigma^{2}$.

Os grupos de contemporâneos foram formados pelo ano de parição $(1988, \ldots, 1996)$ mais a estação de parição, sendo a estação 1 correspondente ao período chuvoso (fevereiro a junho) e a estação 2, ao período seco (julho a janeiro do ano seguinte).

O modelo para análise da DL difere do anterior apenas, por não incluir a covariável duração de lactação.

As estimativas dos componentes de (co)variância necessárias para estimação dos parâmetros genéticos foram obtidas pela metodologia da Máxima Verossimilhança Restrita Livre de Derivadas, sob modelo animal em análise bicaracterística, utilizando o programa MTDFREML, descrito por Boldman et al. (1995). O programa utiliza o algoritmo simplex para localizar o mínimo de $-2 \log _{\mathrm{e}} \mathrm{L}$ ( $\mathrm{L}=$ função de verossimilhança) e os componentes de (co)variância que minimizam $-2 \log _{\mathrm{e}} \mathrm{L}$ são estimativas de máxima verossimilhança, que maximizam a função de verossimilhança.

O critério de convergência utilizado foi a variância dos valores do simplex inferior a $10^{-9}$. Após a convergência, o programa era reiniciado usando as estimativas obtidas anteriormente como valores iniciais. Este procedimento foi repetido até que a diferença entre as estimativas das duas últimas convergências não diferisse. Os valores iniciais foram os obtidos com base na análise de variância efetuada pelo método dos quadrados mínimos.

O modelo de análise pode ser representado, matricialmente, como segue:

$$
Y=X b+Z_{1} d+Z_{2} p+\varepsilon
$$

em que: $Y=$ vetor das observações $\mathrm{n} \times 1 ; X=$ matriz $\mathrm{n} \times \mathrm{f}$ de incidência, relacionando as observações aos efeitos fixos; $b=$ vetor $\mathrm{f} \times 1$ de efeitos fixos; $Z_{1}=$ matriz $\mathrm{n} \times \mathrm{N}$ de incidência, relacionando as observações aos efeitos genéticos aditivos diretos; $d=$ vetor $\mathrm{N} \times 1$ de efeitos genéticos aditivos diretos, $\sim \mathrm{N}\left(0, \mathrm{~A} \sigma_{d}^{2}\right) ; Z_{2}=$ matriz $\mathrm{n} \times \mathrm{N}$ de incidência, relacionando as observações aos efeitos de ambientes permanentes; $p=$ vetor $\mathrm{Nx} 1$ de efeitos de ambientes permanentes, $\sim \mathrm{N}\left(0, \mathrm{I}_{p}^{2}\right) ; \varepsilon=$ vetor $\mathrm{n} \times 1 \mathrm{de}$ efeitos residuais, $\sim \mathrm{N}\left(0, \mathrm{I} \sigma_{e}^{2}\right)$. Sendo: $\mathrm{A}=$ matriz de parentesco; $\mathrm{I}=$ matriz identidade de ordem $\mathrm{n}$; $\sigma_{d}^{2}=$ variância genética aditiva direta; $\sigma_{p}^{2}=$ variância de ambiente permanente; $\sigma_{e}^{2}=$ variância residual; $\mathrm{n}=$ número de observações de produção de leite e duração da lactação; $f$ = número de níveis de efeitos fixos; e $\mathrm{N}$ = número de animais com observação.

Os efeitos fixos e covariáveis considerados neste modelo foram os mesmos utilizados no modelo das análises dos efeitos de ambiente.

\section{Resultados e Discussão}

As médias encontradas para produção de leite e duração de lactação foram, respectivamente, 257,7 \pm $54,7 \mathrm{~kg}$ de leite e $185,5 \pm 46,9$ dias de duração da lactação, resultando em produção média diária de $1,38 \mathrm{~kg}$ de leite. Esse resultado é satisfatório, considerando-se a região onde a fazenda está localizada e o período estudado. 
O resumo da análise de variância para as características estudadas é apresentado na Tabela 1. Todos os efeitos foram significativos $(\mathrm{P}<0,05)$, com exceção do tipo de parto para PL e DL e da idade da cabra ao parto para DL.

A influência de grupo contemporâneo já era esperada, pois as flutuações do clima, especificamente a variação de pluviosidade entre e dentro dos anos estudados causa variações no desempenho por meio da estacionalidade na disponibilidade de alimentos, em termos qualiquantitativos, mesmo com o manejo alimentar utilizado na Fazenda.

A não-significância do tipo de parto sobre as características estudadas pode ser atribuída ao manejo dos cabritos, que são aleitados artificialmente. Dessa forma, fica evidente a falta de estímulo diferenciado, em que as cabras, mesmo de tipos de partos diferentes, passam, após a parição, receber o mesmo estímulo (ordenha), corroborando os relatos de Soares Filho et al. (2001).

O resultado obtido para idade da cabra ao parto em relação à PL é biologicamente correto, uma vez que a cabra aumenta sua produção até certa idade e tende a decrescer, descrevendo uma função quadrática, o que está de acordo com a maioria dos trabalhos encontrados na literatura, como os de Mourad (1992) e Gonçalves et al. (2001). A equação e respectiva curva de regressão da produção de leite, em função da idade ao parto, encontram-se na Figura 1. A idade em que as cabras apresentaram máxima produção foi, aproximadamente, aos 56,7 meses (4,7 anos).

A duração da lactação influenciou a produção de leite de forma quadrática (Tabela 1). A regressão e a respectiva equação são apresentadas na Figura 2 . Houve relação crescente entre produção de leite e duração da lactação. Entretanto, esta relação, acontece até certo limite, evidenciando que lactações mais longas não favorecem maior produção de leite, ou seja, a produção de leite não aumenta linearmente com o avanço do período de lactação.

As estimativas de herdabilidade para PL e DL foram de magnitude média (Tabela 2). A herdabilidade estimada para PL encontra-se dentro do intervalo das estimativas observadas na literatura para cabras de origem tropical, próxima à relatada por Gonçalves et

Tabela 1 - Resumo da análise de variância para produção de leite (PL) e duração da lactação (DL)

Table 1 - Summary of the analysis of variance for milk production (MP) and lactation length (LL)

\begin{tabular}{|c|c|c|c|c|c|c|}
\hline \multirow[t]{2}{*}{$\begin{array}{l}\text { Fontes de variação } \\
\text { Source of variation }\end{array}$} & \multicolumn{3}{|c|}{$\begin{array}{l}\mathrm{PL} \\
M P\end{array}$} & \multicolumn{3}{|c|}{$\begin{array}{l}\mathrm{DL} \\
L L\end{array}$} \\
\hline & $\begin{array}{l}\text { GL } \\
D F\end{array}$ & $\begin{array}{l}\text { Quadrado médio } \\
\text { Mean square }\end{array}$ & $\begin{array}{l}\text { P-Valor } \\
P \text { - Value }\end{array}$ & $\begin{array}{l}\mathrm{G} \\
D F\end{array}$ & $\begin{array}{l}\text { Quadrado médio } \\
\text { Mean square }\end{array}$ & $\begin{array}{l}\text { P-Valor } \\
P \text { - Value }\end{array}$ \\
\hline $\begin{array}{l}\text { Bode } \\
\text { Buck }\end{array}$ & 17 & $10.203,8$ & 0,0001 & 17 & $4.623,2$ & 0,005 \\
\hline $\begin{array}{l}\text { Grupo contemporâneo } \\
\text { Contemporary group }\end{array}$ & 17 & $27.645,8$ & 0,0001 & 17 & $16.838,8$ & 0,0001 \\
\hline $\begin{array}{l}\text { Tipo de parto } \\
\text { Kidding type } \\
\text { Idade da cabra ao partc } \\
\text { Age of goat at kidding }\end{array}$ & 1 & $1.566,7$ & 0,4698 & 1 & 388,1 & 0,674 \\
\hline $\begin{array}{l}\text { Regressão linear } \\
\text { Linear regression }\end{array}$ & 1 & $176.253,1$ & 0,0001 & 1 & $7.673,3$ & 0,0621 \\
\hline $\begin{array}{l}\text { Regressão quadrática } \\
\text { Quadratic regression } \\
\text { Duração da lactação } \\
\text { Lactation length }\end{array}$ & 1 & $131.177,8$ & 0,0001 & 1 & $4.565,8$ & 0,1499 \\
\hline $\begin{array}{l}\text { Regressão linear } \\
\text { Linear regression }\end{array}$ & 1 & $356.276,2$ & 0,0001 & - & - & - \\
\hline $\begin{array}{l}\text { Regressão quadrática } \\
\text { Quadratic regression }\end{array}$ & 1 & $99.937,2$ & 0,0001 & - & & - \\
\hline $\begin{array}{l}\text { Resíduo } \\
\text { Error }\end{array}$ & 649 & $\begin{array}{r}2995,6 \\
\mathrm{CV}=21,2 \%\end{array}$ & - & 651 & $\begin{array}{c}2.196,9 \\
\mathrm{CV}=25,2 \%\end{array}$ & - \\
\hline
\end{tabular}

$\mathrm{P}-$ valor $=$ valor de probabilidade.

$P-$ Value $=$ probability of value.

R. Bras. Zootec., v.33, n.6, p.1426-1431, 2004 


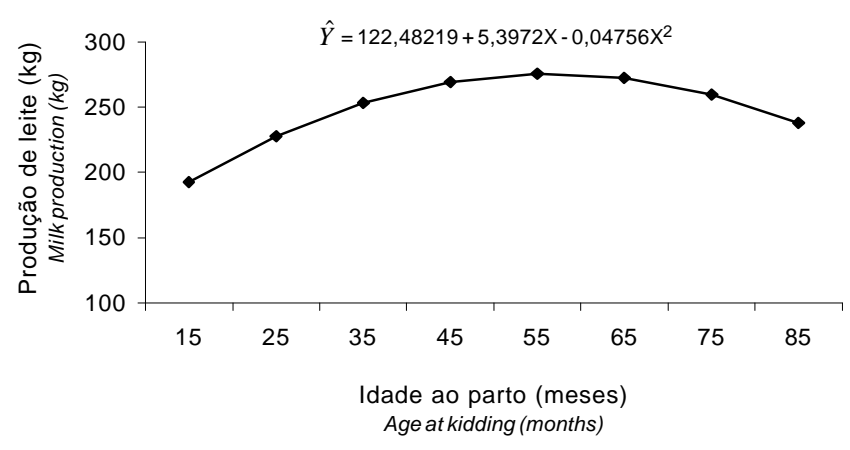

Figura 1 - Regressão da produção de leite, em função da idade ao parto.

Figure 1 - Regression of the milk production, in function of age at kidding.

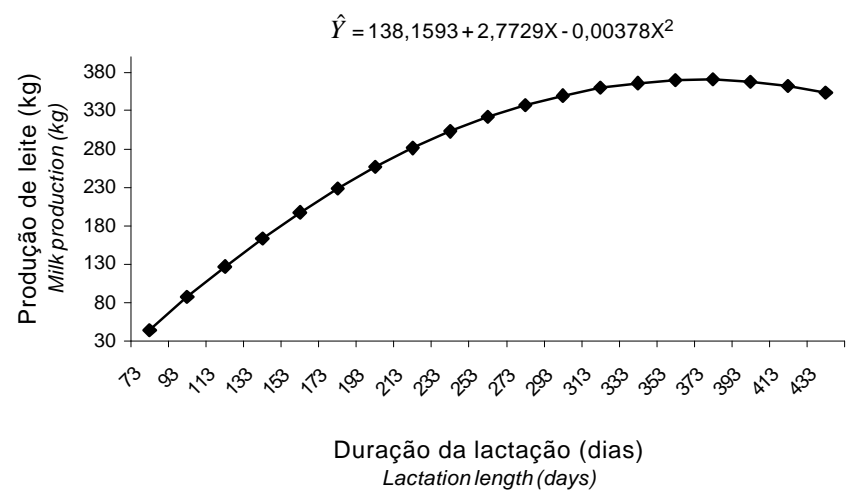

Figura 2 - Regressão da produção de leite, em função da duração da lactação.

Figure 2 - Regression of the milk production, in function of lactation length.

al. (2001) e superior às encontradas por Soares Filho et al. (2001). A estimativa de herdabilidade para DL encontra-se acima dos valores obtidos para cabras especializadas, criadas em condições brasileiras. Essas estimativas de herdabilidade indicam correlação média entre o fenótipo e genótipo dos indivíduos. Portanto, a seleção de fêmeas pelo fenótipo promoverá lento incremento genético na população. Assim, a partir dos resultados obtidos e pelo fato de as características serem limitadas ao sexo, para os reprodutores, o teste de progênie é o método indicado como o mais eficaz para seleção dos animais destinados à reprodução.

A seleção para DL só seria interessante em programas de melhoramento se a meta almejada fosse maximizar a eficiência produtiva e reprodutiva do rebanho. Assim, a seleção seria praticada com base nas cabras que apresentassem maior produção em menor período de lactação possível. Como foi constatado neste estudo, a relação entre DL e PL não é linear, porém a relação entre DL e intervalo de parto, constatada por Sarmento et al. (2003), é linear, ou seja, cada dia a mais na DL correspondeu a um aumento de 0,59 dias no intervalo de parto. Dessa forma, a seleção de cabras com maiores períodos lactantes refletirá em maiores intervalos de partos, conseqüentemente menor pressão de seleção e ganho genético anual.

As estimativas de repetibilidade para as características estudadas foram de média magnitude (Tabela 2), de modo que uma única medida no animal pode não representar a real capacidade de produção, sendo necessário, portanto, mais de uma medida para aumentar a acurácia das estimativas. Estimativas inferiores foram registradas por Ribeiro (1997), com cabras de raças especializadas no Brasil, para ambas as características.

A correlação genética estimada entre PL e DL foi alta (Tabela 2), indicando que grande parte dos efeitos genéticos aditivos age sinergicamente em ambas as características, resultado que sugere que a seleção de cabras com maior produção de leite levaria a lactações mais longas. Entretanto, a resposta correlacionada não depende só da associação genética entre as características, como também das herdabilidades das características. Dessa forma, a resposta correlacionada pela seleção indireta, embora eficiente, em decorrência da alta correlação genética, resultará em baixos ganhos genéticos. A correlação estimada neste estudo encontra-se próxima às verificadas por Kennedy et al. (1982) e Gonçalves (1996), em cabras de raças exóticas.

Tabela 2 - Componentes de variância genética adițva $\left(\sigma_{d}^{2}\right)$, de ambiente permanente $\left(\sigma_{p}^{2}\right)$, herdabilidade $\left(\mathrm{h}^{2}\right)$, repetibilidade $(\mathrm{t})$ e correlação genética entre PL e DL

Table 2 - Additive genetic of variance components $\left(\sigma_{d}^{2}\right)$ of permanent environment $\left(\sigma_{p}^{2}\right)$ heritability $\left(h^{2}\right)$, repeatability $(t)$ and genetic correlation between $M P$ and $L L$

\begin{tabular}{lccccc}
\hline $\begin{array}{l}\text { Variáveis } \\
\text { Variables }\end{array}$ & $\sigma_{d}^{2}$ & $\sigma_{p}^{2}$ & $h^{2}$ & $t$ & $r_{g}$ \\
\hline $\mathrm{PL}$ & 2206,26 & 1153,06 & 0,23 & 0,35 & 0,96 \\
$M P$ & 568,87 & 11,29 & 0,20 & 0,24 & \\
$\mathrm{DL}$ & & & & & \\
$L L$ & & & & & \\
\hline
\end{tabular}

\section{R. Bras. Zootec., v.33, n.6, p.1426-1431, 2004}




\section{Conclusões}

Melhorias no manejo alimentar devem ser implementadas, principalmente no que tange ao armazenamento de forragem, visando diminuir os efeitos indiretos do clima.

Em razão de as herdabilidades das características PL e DL serem de média magnitude e por estas serem limitadas ao sexo, indica-se o teste de progênie como o método mais eficaz para seleção de bodes.

Apesar da alta correlação estimada entre produção de leite e duração da lactação, o ganho genético pela seleção indireta é pequeno, pois as herdabilidades das características são relativamente baixas.

\section{Literatura Citada}

BOLDMAN, K.G.; KRIESE, L.A.; Van VLECK, D.L. et al. A manual for use of MTDFREML A set of programs to obtain estimates of variances and covariances [DRAFT] Lincon: USDA/ARS, 1995. 120p.

CONSTANTINOU, A.; BEUING, R.; MAVROGENIS, A.P. Genetic and phenotypic parameters for some reproductive and milk prodution characters of the Damascus goat. Zeitschrift fur Tierzuchtung und Zuchtungsbiologie, v.102, p.301-307, 1985.

CORDEIRO, P.R.C. Produção de leite d cabra no Brasil. In: REUNIÃO ANUAL DA SOCIEDADE BRASILEIRA DE ZOOTECNIA, 38., 2001, Piracicaba. Anais... Piracicaba: Sociedade Brasileira de Zootecnia, 2001. p.497.

GONÇALVES, H.C.; SILVA, M.A.; WECHSLER, F.S. et al. Fatores genéticos e de meio na produção de leite de caprinos leiteiros. Revista Brasileira de Zootecnia, v.30, n.3, p.719-729, 2001.

GONÇALVES, H.C. Fatores genéticos e de meio em algumas características produtivas e reprodutivas de caprinos. Viçosa, MG: Universidade Federal de Viçosa, 1996. 141p. Tese (Doutorado em Zootecnia) - Universidade Federal de Viçosa, 1996.
KENNEDY, B.W.; FINLEY, C.M.; BRADFORD, G.E. Phenotypic and genetic relationships between reproduction and milk production in dairy goats. Journal of Dairy Science, v.65, p.2373-83, 1982.

MAVROGENiS, A.P.; CONSTATINOU, A.; LOUCA, A. Environmental and genetic causes of variation in production traits of Damascus goats. Animal Production Science, v.33, p.99-104, 1984.

MOURAD, M. Effects of month of kidding, parity and litter size on milk yield of Alpine goats in Egypt. Small Ruminants Research, v.8, p.545-554, 1992.

PIMENTA FILHO, E.C.; RIBEIRO, M.N.; SOUSA, W.H. Melhoramento genético de pequenos ruminantes para carne e leite. In: CONGRESSO NORDESTINO DE PRODUÇÃO ANIMAL, 2., 2000, Teresina. Anais... Teresina: Sociedade Nordestina de Produção Animal, 2000. p.107-116.

RIBEIRO, A.C. Estudo dos efeitos genéticos e de ambiente sobre características de importância econômica em caprinos da raça Saanen. Jaboticabal: Universidade Estadual Paulista, 1997. 85p. Dissertação (Mestrado em Zootecnia) - Universidade Estadual Paulista, 1997.

SARMENTO, J.L.R.; PIMENTA FILHO, E.C.; RIBEIRO, M.N. et al. Fatores genéticos e ambientais sobre o intervalo de partos de cabras leiteiras no semi-árido nordestino. Revista Brasileira de Zootecnia, v.32, n.4, p.875-879, 2003.

STATISTICAL ANALYSIS SYSTEM - SAS. User's guide: Statistics. Version 8.0, Cary: 1999.

SOARES FILHO, G.; MCMANUS, C.; MARIANTE, A.S. Fatores genéticos e ambientais que influenciam algumas características de reprodução e produção de leite em cabras no Distrito Federal. Revista Brasileira de Zootecnia, v.30, n.1, p.53-59, 2001.

Recebido em: 09/12/02

Aceito em: 17/03/03 